\title{
CHEMOGENETIC CONTROL OF NANOBODIES
}

2

3

4 Helen Farrants ${ }^{1,2}$, Miroslaw Tarnawski ${ }^{3}$, Thorsten G. Müller ${ }^{4}$, Shotaro Otsuka ${ }^{5,6}$, Julien

5 Hiblot ${ }^{1}$, Birgit Koch ${ }^{1}$, Moritz Kueblbeck ${ }^{5}$, Hans-Georg Kräusslich4, Jan Ellenberg ${ }^{5}$, Kai

6 Johnsson ${ }^{1,2, *}$

7

81 Department of Chemical Biology, Max Planck Institute for Medical Research, Jahnstrasse 29, 69120

9 Heidelberg, Germany

102 Institute of Chemical Sciences and Engineering, École Polytechnique Fédérale de Lausanne (EPFL),

111015 Lausanne, Switzerland

123 Protein Expression and Characterization Facility, Max Planck Institute for Medical Research,

13 Jahnstrasse 29, 69120 Heidelberg, Germany

$14{ }^{4}$ Department of Infectious Diseases, Virology, University Hospital Heidelberg, Im Neuenheimer Feld 15344,69120 Heidelberg, Germany

165 Cell Biology and Biophysics Unit, European Molecular Biology Laboratory (EMBL), Meyerhofstrasse

17 1, 69117 Heidelberg, Germany

186 Current address: Max Perutz Labs, a joint venture of the University of Vienna and the Medical 19 University of Vienna, Dr. Bohr-Gasse 9, 1030 Vienna, Austria

Email: johnsson@mr.mpg.de

\section{ABSTRACT}

24 We introduce an engineered nanobody whose affinity to green fluorescent protein (GFP) can be switched on and off with small molecules. By controlling the cellular localization of GFP fusion proteins, the engineered nanobody allows to study their role in basic biological processes, an approach that should be applicable to numerous previously described GFP fusions. We also outline how the binding affinities of other nanobodies can be controlled by small molecules. 


\section{MAIN TEXT}

32 The variable domains of heavy chain-only antibodies ${ }^{1}$, commonly abbreviated as nanobodies, are powerful tools to interrogate processes in living systems.

Nanobodies can be selected to bind to a variety of targets with high affinity and selectivity, and can be functionally expressed inside cells ${ }^{2,3}$. The range of applications of nanobodies would be greatly expanded if their binding affinity towards their target could be rapidly switched on and off with a cell-permeable and non-toxic molecule. Proteins such as kinases and Cas9 have been engineered to control their activity with small molecules ${ }^{4-6}$, but these approaches have not been applied to nanobodies. Here, we introduce "ligand-modulated antibody fragments" (LAMAs), which combine the high selectivity and specificity of nanobodies with the fast temporal control offered through the use of small molecules. LAMAs are generated by inserting a circularly permutated bacterial dihydrofolate reductase (cpDHFR) ${ }^{7}$ into nanobodies. The new termini of this cpDHFR are located in an active site loop of wild-type DHFR. Furthermore, cpDHFR is partially unfolded in the absence of its cofactor nicotinamide adenine dinucleotide phosphate (NADPH) and DHFR inhibitors such as trimethoprim (TMP) ${ }^{8,9}$. TMP is a clinically approved anti-bacterial drug that has excellent cell and tissue permeability and is not toxic for mammalian cells. LAMAs disrupt the binding of the nanobody to its target by exploiting the change in conformation of cpDHFR upon binding of NADPH and DHFR inhibitors (Fig. 1a). The

51 first nanobody we subjected to this approach was the enhancer nanobody for GFP10.

52 Specifically, we inserted cpDHFR into various sites of the enhancer nanobody and measured the binding affinities of the protein chimeras to wild-type GFP (wtGFP) in the presence and absence of the ligands NADPH and TMP (Fig. 1b and 
Supplementary Fig. 1). The most promising insertion hits were in the

56

57 complementary-determining region 3 (CDR3), which is often essential for making high affinity contacts between nanobodies and their targets ${ }^{11}$. Of these hits, we analyzed GFPLAMAF98, GFPLAMAG97, and GFPLAMAN95 in greater detail (Fig. 1C-e and Supplementary Fig. 2). All three GFPLAMAs retained a single-digit nanomolar affinity to GFP in the absence of ligands. For all three nanobodies, the affinity towards GFP was dramatically decreased in the presence of NADPH and TMP such that no binding to GFP could be detected for GFPLAMAF98 and GFPLAMAG97 (Fig. 1c-e and Supplementary Fig. 3). For GFPLAMAf98 the presence of NADPH alone also affected the binding affinity to GFP, whereas the affinity of GFPLAMAG97 and GFPLAMAN95 was not affected by NADPH.

The kinetics of dissociation of the complexes between GFP and GFPLAMAF98 or GFPLAMAG97 upon addition of TMP were on the timescale of minutes: $t_{1 / 2}=34 \pm 1 \mathrm{sec}$ and $t_{1 / 2}=5.6 \pm 0.5 \mathrm{~min}$ for GFPLAMAF98 and GFPLAMAG97, respectively (Fig. 1f,g). Subsequent removal of TMP by addition of wild-type DHFR resulted in reformation of the complexes within minutes (Fig. 1f,g). The complex could then be dissociated again by addition of an excess amount of TMP (Fig. 1f,g). The dissociation kinetics of the complexes could also be tuned using DHFR inhibitors with different affinities to DHFR (Supplementary Fig. 4). These experiments underline that GFPLAMAF98 and GFPLAMAG97 can be repeatedly switched on and off through the addition of DHFR inhibitors. 
To understand how the cpDHFR insertion into nanobodies allowed control of binding affinities, we solved the crystal structures of GFPLMAF98 and GFPLAMAG97 in complex with NADPH and TMP. No major structural changes were seen in the nanobody domain of the two LAMAs relative to enhancer nanobody. Comparing these structures with the structure of enhancer nanobody bound to GFP suggests that folded cpDHFR sterically hampers binding to GFP (Fig. 1h and Supplementary Fig. 5). The TMP-dependent control of the GFPLAMAs was abolished when GGS-linkers were inserted between cpDHFR and the nanobody (Supplementary Fig. 6), indicating that the switching of nanobody affinity did not solely arise from insertion of the protein domain.

Given the large number of nanobodies that have been selected and characterised ${ }^{12}$, we attempted to expand the LAMA concept to other targets. Nanobodies for Gassociated kinase ${ }^{13}$, and for lamina-associated polypeptide $1^{14}$, did not allow for cpDHFR insertion into the tried positions (Supplementary Fig. 7a,b). The minimizer nanobody for GFP ${ }^{10}$ greatly decreased its affinity to GFP on cpDHFR insertion, but responded to the addition of ligands (Supplementary Fig. 7c). A nanobody for the C-terminal region of the p24 HIV capsid protein (manuscript in preparation) could be readily converted into a LAMA on insertion of cpDHFR into the CDR3 loop (Fig. 1i and Supplementary Fig. 8). The ${ }^{\mathrm{p} 24}$ LAMAs98 showed low nanomolar affinity for p24 HIV capsid protein when no ligands were present. Neither TMP nor NADPH alone could decrease the affinity of ${ }^{\text {P24 }}{ }^{2 A M A s 98}$ for its target, but addition of both ligands reduced the affinity 70 -fold (Fig. 1j). These experiments highlight the transferability for the LAMA approach to other nanobodies. 
103 The binding of both the ${ }^{\text {24 }}$ LAMA and GFPLAMAs to their targets could be switched on

104 and off through the addition of TMP in live cells (Fig 2). Intracellular NADPH concentration in live cells is estimated to be $3.1 \pm 0.3 \mu \mathrm{M}^{15}$, thus providing a basal level of NADPH. The expression of the cytosolic p24 precursor polyprotein Gag in HIV transfected cells stably expressing an EGFP_p24LAMAs98 fusion resulted in sequestering of the LAMA in the cytosol (Fig. 2a). However, the LAMA was released from the p24 domain of Gag by the addition of TMP within minutes, as demonstrated by diffusion of EGFP_p24LAMAs98 into the nucleus (Fig. 2a and Supplementary Fig. 9). Targeting GFPLAMAs to the inner leaflet of the plasma membrane by a Lyn kinase derived sequence ${ }^{16}$ (Lyn-GFPLAMA) resulted in localization of EGFP to the plasma membrane, which could be released into the cytosol through addition of TMP (Fig.

2b and Supplementary Fig. 10a). Similarly, targeting GFPLAMAs to the outer membrane of mitochondria ${ }^{17}$ (mito-GFPLAMAs) resulted in reversible sequestering of EGFP to the outer membrane of mitochondria (Fig. 2c and Supplementary Fig. 10b

117 ). The kinetics of the TMP-dependent release and sequestering of EGFP from the outer mitochondrial membrane was evaluated by following the appearance and disappearance of the fluorescence of nuclear EGFP (Fig. 2d,e and Supplementary

120 Fig. 11). The release and sequestering of EGFP upon addition and wash-out of TMP occurred on a timescale of minutes, and could be repeated over several cycles (Fig. 
GFPLAMAs can be used to control the localization of other family members of GFP.

127 Shadow $\mathrm{G}^{18}$, a non-fluorescent version of GFP (Fig. $2 f$ and Supplementary Fig.

128 12). The high affinity of GFPLAMAs for GFP also allows to mislocalize GFP fusion

129 proteins that are part of larger protein complexes. For example, transient transfection

130 of mito-GFPLAMAF98 into a genome-edited cell line expressing NUP62-mEGFP

131 (Supplementary Fig. 13a), a component of the nuclear pore complex, resulted in

132 sequestering of NUP62-mEGFP from the nuclear envelope to the mitochondria in the absence of TMP (Fig $\mathbf{2 g}$ and Supplementary Fig. 13b). Upon addition of TMP, NUP62-mEGFP localized to the nuclear membrane.

GFP fusion proteins are omnipresent in the life sciences and our GFPLAMAs offer a new way to probe the function of these proteins. To demonstrate the potential of GFPLAMAs for mechanistic studies, we used mito-GFPLAMAF98 to control the function of a GFP fusion of Mad2L1, an important component of the mitotic checkpoint complex (Fig $2 \mathbf{h}$ and Supplementary Fig. 14). Knock-down of Mad2L1 reduces mitotic duration and increases the percentage of polylobed nuclei ${ }^{19}$. A HeLa Kyoto cell line in which endogenous Mad2L1 has been tagged with EGFP has been previously described and used to map the localizations of Mad2L1 during mitosis ${ }^{20}$.

144 We stably expressed mito-GFPLAMAF98 in the Mad2L1-EGFP cell line, and observed

145 how sequestering Mad2L1-EGFP to the mitochondria affected the outcome of cell division (Supplementary Fig. 15 and Supplementary Video 1-4). In the absence of

147 TMP, we observed an increase in the percentage of polylobed nuclei following

148 mitotic events relative to cells not expressing mito-GFPLAMAF98 (85 $\pm 13 \%$ vs. $10 \pm 6$ 
\%; Fig. 2i and Supplementary Fig. 16). Addition of TMP to cells expressing mito-

GFPLAMA $_{\text {F98 }}$ reduced the levels to those not expressing mito-GFPLAMAF98 $(10 \pm 11$

\%). Next, nocodazole, a small molecule which prevents attachment of microtubules

to kinetochores, was added to activate the mitotic checkpoint complex. After

treatment with nocodazole, cells in which Mad2L1-EGFP had been sequestered at

154 the mitochondria were able to override mitotic arrest whereas treatment with

nocodazole and TMP lead to mitotic arrest, as expected (Fig. 2j and

Supplementary Fig 17). These data show that the function of Mad2L1-EGFP in the mitotic checkpoint complex can be controlled through its TMP-dependent interaction with mito-GFPLAMAF98.

In summary, LAMAs are a generally applicable chemogenetic tool to reversibly

control the location and function of proteins, including the most commonly used class of fusion proteins (GFP). This tool opens up countless applications in research to study basic biological questions. As TMP is a clinically approved drug the approach might also be applicable in vivo. Furthermore, the design principle introduced here should be applicable for the generation of other switchable proteins.

\section{Acknowledgments}

168 This work was supported by the Max Planck Society, the École Polytechnique

169 Fédérale de Lausanne, the NCCR Chemical Biology. Research in the Kräusslich

170 group was supported by the Deutsche Forschungsgemeinschaft (DFG, German

171 Research Foundation) - Projektnummer 240245660 - SFB 1129 project 5 (H.-G.K).

172 Research in the Ellenberg group was supported by the Paul G. Allen Frontiers Group 
173 through an Allen Distinguished Investigators Grant to J.E., the National Institutes of

174 Health Common Fund 4D Nucleome Program (Grant U01 EB021223 / U01

175 DA047728 to J.E.) and the European Molecular Biology Laboratory (EMBL; S.O.,

176 M.K., J.E.). The authors thank Ilme Schlichting for X-ray data collection. Diffraction

177 data were collected at the Swiss Light Source, beamline X10SA, of the Paul

178 Scherrer Institute, Villigen, Switzerland. The authors would like to thank Luc

179 Reymond, Johannes Broichhagen and Bettina Mathes for providing reagents. The

180 authors would like to thank Manuel Eguren for valuable discussions.

\section{Additional Information}

183 Requests for reagents and plasmids should be directed to Kai Johnsson. All

184 requests for the Nup62-mEGFP cell line should be directed to Jan Ellenberg.

\section{Author Contributions}

H.F. and K.J. designed the study. H.F generated, characterized and applied all

LAMAs. M.T. solved the crystal structures of GFPLAMAs. J.H. helped analyse the crystal structures. M.K. generated the NUP62-mEGFP cell line and S.O. performed the NUP62-mEGFP translocation experiments. B.K. helped with generation of stable cell lines with LAMAs. T.G.M. generated stable cells lines of ${ }^{224}{ }^{2}$ AMA and the manuscript with input from all authors.

196 The authors declare no competing interests. 
bioRxiv preprint doi: https://doi.org/10.1101/683557; this version posted June 27, 2019. The copyright holder for this preprint (which was

not certified by peer review) is the author/funder, who has granted bioRxiv a license to display the preprint in perpetuity. It is made available under aCC-BY-ND 4.0 International license. 


\section{References}

2011 Hamers-Casterman, C. et al. Naturally occurring antibodies devoid of light chains. Nature 363, 446-448, (1993).

204

2 Muyldermans, S. Nanobodies: natural single-domain antibodies. Annu. Rev. Biochem. 82, 775-797, (2013).

206

3 Ingram, J. R., Schmidt, F. I. \& Ploegh, H. L. Exploiting nanobodies' singular traits. Annu. Rev. Immunol. 36, 695-715, (2018).

4 Stein, V. \& Alexandrov, K. Synthetic protein switches: design principles and applications. Trends Biotechnol. 33, 101-110, (2015).

Oakes, B. L. et al. Profiling of engineering hotspots identifies an allosteric CRISPR-Cas9 switch. Nat. Biotechnol. 34, 646-651, (2016). Karginov, A. V., Ding, F., Kota, P., Dokholyan, N. V. \& Hahn, K. M. Engineered allosteric activation of kinases in living cells. Nat. Biotechnol. 28, 743, (2010). Eng. 11, 707-713 (1998).

$8 \mathrm{Yu}, \mathrm{Q}$. et al. Semisynthetic sensor proteins enable metabolic assays at the point of care. Science 361, 1122, (2018).

9 Nakamura, T. \& Iwakura, M. Circular permutation analysis as a method for distinction of functional elements in the M20 Loop of Escherichia coli dihydrofolate reductase. J. Biol. Chem. 274, 19041-19047 (1999).

10 Kirchhofer, A. et al. Modulation of protein properties in living cells using nanobodies. Nat. Struct. Mol. Biol. 17, 133-138, (2009).

11 De Genst, E. et al. Molecular basis for the preferential cleft recognition by dromedary heavy-chain antibodies. Proc. Natl. Acad. Sci. USA 103, 4586-4591, (2006).

12 Wilton, E. E., Opyr, M. P., Kailasam, S., Kothe, R. F. \& Wieden, H.-J. sdAb-DB: The Single Domain Antibody Database. ACS Synth. Biol. 7, 2480-2484, (2018).

13 Chaikuad, A. et al. Structure of cyclin G-associated kinase (GAK) trapped in different conformations using nanobodies. Biochem. J. 459, 59-69, (2014).

14 Sosa, B. A. et al. How lamina-associated polypeptide 1 (LAP1) activates Torsin. elife 3, e03239, (2014).

15 Tao, R. et al. Genetically encoded fluorescent sensors reveal dynamic regulation of NADPH metabolism. Nat. Methods 14, 720-728, (2017).

16 Inoue, T., Heo, W. D., Grimley, J. S., Wandless, T. J. \& Meyer, T. An inducible translocation strategy to rapidly activate and inhibit small GTPase signaling pathways. Nat. Methods 2, (2005).

17 Kanaji, S., Iwahashi, J., Kida, Y., Sakaguchi, M. \& Mihara, K. Characterization of the signal that directs Tom20 to the mitochondrial outer membrane. J. Cell Biol. 151, 277-288, (2000).

Murakoshi, H., Shibata, A. C. E., Nakahata, Y. \& Nabekura, J. A dark green fluorescent protein as an acceptor for measurement of Förster resonance energy transfer. Sci. Rep. 5, 15334, (2015). throughput live cell imaging. Nat. Methods 7, 747-754, (2010). 
bioRxiv preprint doi: https://doi.org/10.1101/683557; this version posted June 27, 2019. The copyright holder for this preprint (which was

not certified by peer review) is the author/funder, who has granted bioRxiv a license to display the preprint in perpetuity. It is made available under aCC-BY-ND 4.0 International license.

20 Cai, Y. et al. Experimental and computational framework for a dynamic protein atlas of human cell division. Nature 561, 411-415, (2018). 
a

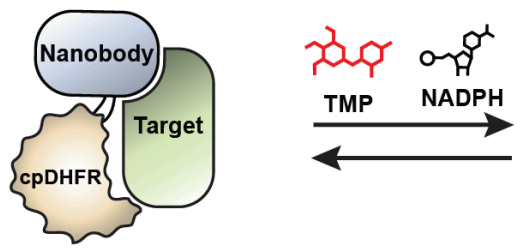

b

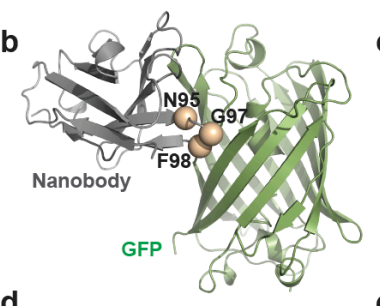

d

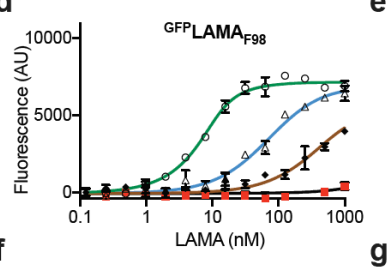

f

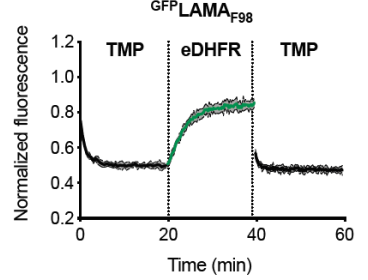

c

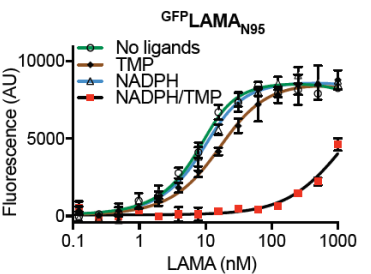

e
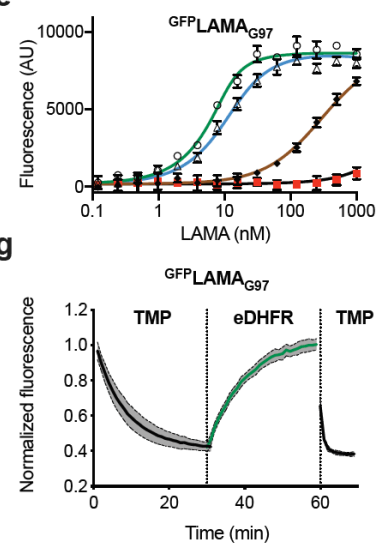

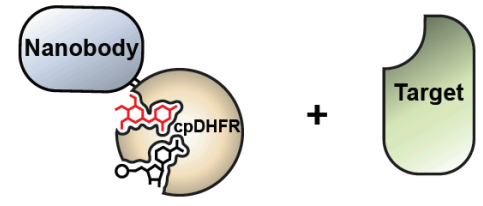

h
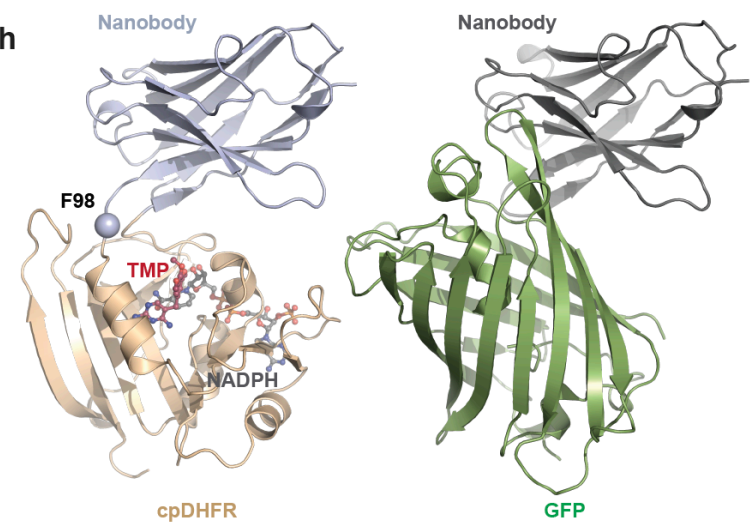

i
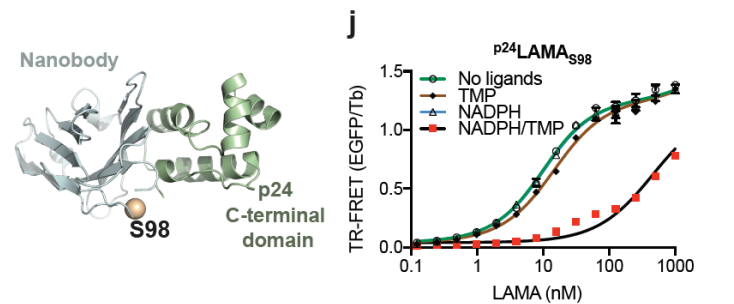

Figure 1. Generation of LAMAs from nanobodies and cpDHFR. (a) Schematic illustration of the design principle of LAMAs. (b) LAMA insertion positions of cpDHFR highlighted as beige spheres mapped onto the structure of the enhancer nanobody bound to GFP, (PDBID = 3K1K). (c-e) Modulation of wtGFP fluorescent emission by GFPLAMA $_{\text {F98 }}$ (c), GFPLAMAG97 (d), GFPLAMAN95 (e), in the presence of NADPH $(100 \mu \mathrm{M})$ and/or TMP $(500 \mu \mathrm{M})$. Mean \pm s.d.. $(\mathbf{f}, \mathbf{g})$ Dissociation kinetics in the presence of NADPH $(100 \mu \mathrm{M})$ of GFPLAMAF98 $(\mathbf{f})$, GFPLAMAG97 $(\mathbf{g})$, from wtGFP measured by wtGFP emission, on the addition of TMP $(1 \mu \mathrm{M})$, followed by the competitive removal by eDHFR $(8 \mu \mathrm{M})$ and addition of excess TMP $(50 \mu \mathrm{M})$. Mean (solid line) + s.d. (grey area). (h) Comparison of the X-ray structure of GFPLAMAF98 in the presence of NADPH and TMP (PDBID = 6RUL), with the enhancer nanobody bound to GFP (PDBID = $3 \mathrm{~K} 1 \mathrm{~K})$. The insertion site $\mathrm{F} 98$ is highlighted as a blue sphere. (i) LAMA insertion position of cpDHFR highlighted as a beige sphere mapped onto the structure of the nanobody for p24, (PDBID = 2XV6). (j) Titration of EGFP-p24LAMAs98 against Tb- 
bioRxiv preprint doi: https://doi.org/10.1101/683557; this version posted June 27, 2019. The copyright holder for this preprint (which was

not certified by peer review) is the author/funder, who has granted bioRxiv a license to display the preprint in perpetuity. It is made available under aCC-BY-ND 4.0 International license.

265 labeled-p24 in a TR-FRET assay in the presence of NADPH (100 $\mu \mathrm{M})$ and/or TMP $266(500 \mu \mathrm{M})$. Mean \pm s.d. 
FIGURE 2

a

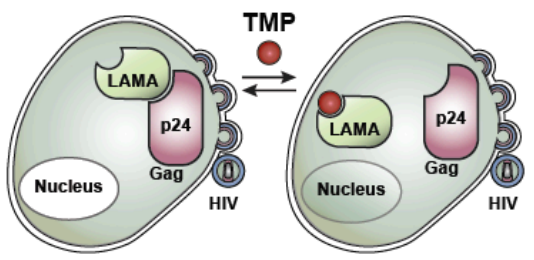

No TMP

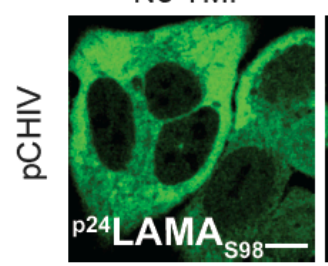

C
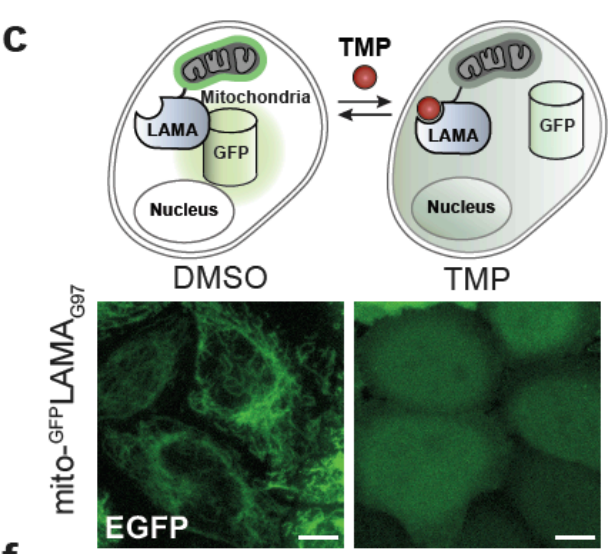

f
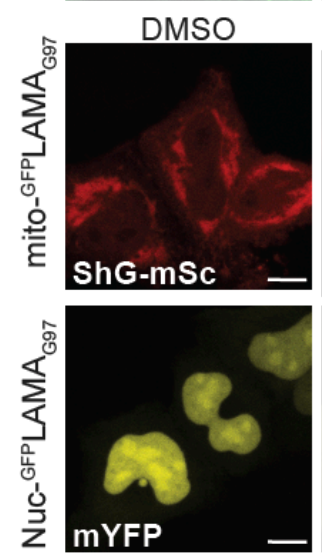

h
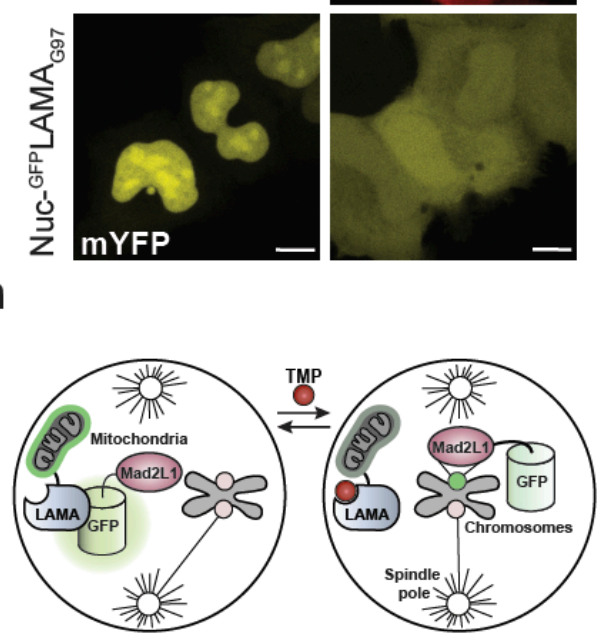

b

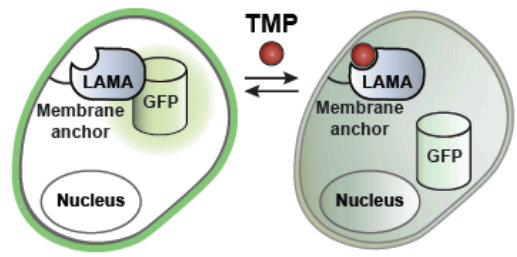

DMSO

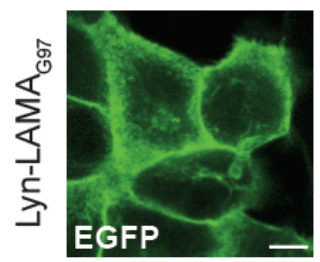

TMP

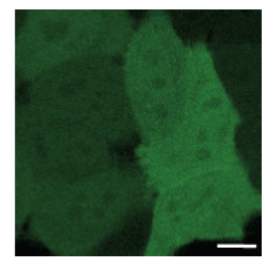

d

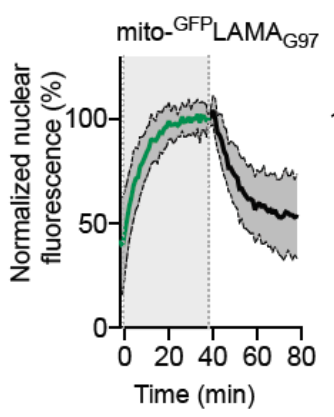

e

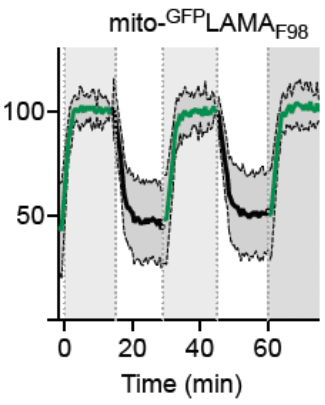

g

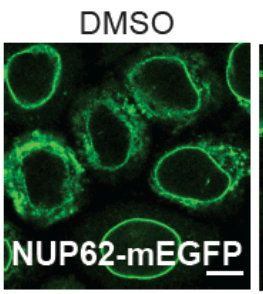

TMP
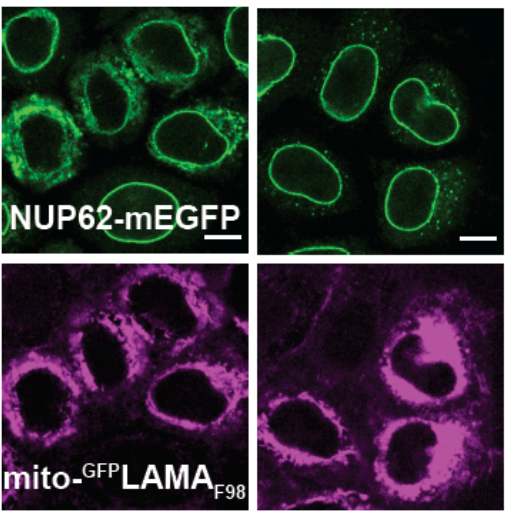

i

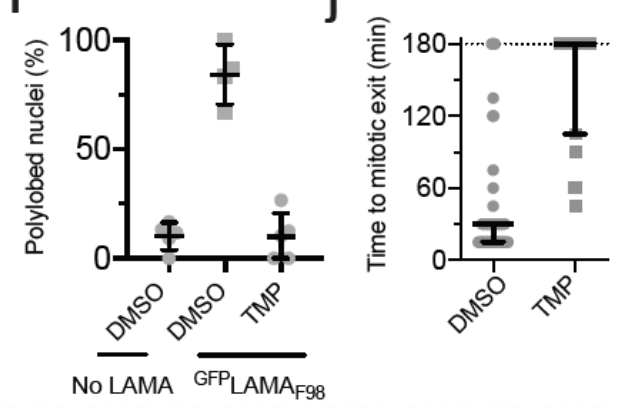

Figure 2. Sequestering and release of protein localization in live cells using LAMAs.

271 (a) Schematic illustration and live-cell imaging of sequestering and release of EGFP- 


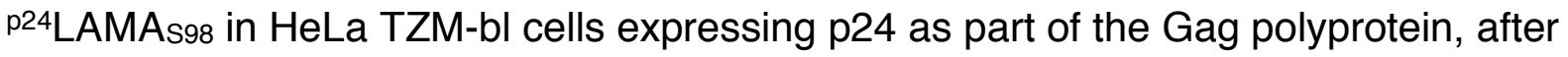

273 transfection with pCHIV. TMP was added to the cells and followed for 40 minutes.

274 (b,c) Schematic illustration and live-cell imaging of HeLa Kyoto cells coexpressing

275 Lyn-GFPLAMAG97 (b) or mito-GFPLAMAG97 (c) with EGFP. Cells were either incubated

276 with DMSO or TMP before imaging. (d,e) Kinetics of release and sequestering of

277 EGFP from mito-GPFLAMAs in U-2 OS cells. EGFP fluorescence in the nucleus was

278 quantified in cells coexpressing EGFP and (d) mito-GPFLAMAG97 ( $\mathrm{N}=26$ cells) or (e)

279 mito-GPFLAMAF98 $(\mathrm{N}=26$ cells). Mean (solid line) \pm s.d. (grey area). TMP was present

280 in greyed areas. (f) Sequestering and release of ShadowG-mScarlet using mito-

281 GFPLAMAG97 and mYFP using nuc-GFPLAMAG97 in HeLa Kyoto cells incubated in

282 DMSO or TMP before imaging. (g) Genome edited NUP62-mEGFP HeLa cells

283 transiently expressing mito-GPFLAMAF98 labeled by BG-TMR via a SNAP-tag fusion.

284 Cells were treated with DMSO or TMP before imaging. (h) Schematic illustration of

285 Mad2L1-EGFP sequestered away from the centromere to the mitochondria by a 286 GFPLAMA.(i, j) Nuclear morphology (i) and duration of mitotic events (j) during live-cell 287 imaging of Mad2L1-EGFP cells stably expressing mito-GPFLAMAF98, after the wash out 288 of TMP $(50 \mu \mathrm{M})$. Percentage polylobed cells (mean \pm s.d., $\mathrm{N}=5$ independent 289 experiments). Duration of mitotic events recorded in the presence of mitotic arrest drug 290 nocodazole (330 nM) (median \pm interquartile range, $\mathrm{N}=63$ and 19 cells, from 3 291 independent experiments). TMP $=10 \mu \mathrm{M}$, unless otherwise stated. Scale bars, $10 \mu \mathrm{m}$. 


\section{ONLINE METHODS}

\section{DNA plasmids and molecular cloning}

295 Plasmids were generated using standard molecular biology techniques. All subcloned sequences were verified using Sanger sequencing, assisted by Geneious software (Biomatters). pCHIV is a non-infectious HIV-1 viral construct lacking LTRs and the nef gene $^{21}$. pCHIV env(stop) was described earlier ${ }^{22}$ and contains a Klenow Polymerase fill in at the Ndel site to generate a frameshift within the env gene. mCherry was inserted between MA and CA of the gag polyprotein and was described earlier ${ }^{23}$. pWPI puro was obtained from Oliver Fackler ${ }^{24}$. psPAX2 was a gift from Didier Trono (Addgene plasmid \#12260) and pCMV-VSV-G was a gift from Bob Weinberg (Addgene plasmid \#8454).

\section{Chemical Reagents}

306 Trimethoprim (TMP), pyrimethamine (Pyr) and methotrexate (MTX) were purchased

307 from Sigma Aldrich. Stock solutions of $50 \mathrm{mM}$ TMP in DMSO were used for both in 308 vitro and in cell analysis. NADPH was purchased from PanReac Biochem, and stocks made to $10 \mathrm{mM}$ fresh in aqueous buffer and used at the indicated concentrations. Nocodazol and reversine were purchased from Selleckchem. DMSO stock solutions were made fresh before use. Fluorescent dyes for live-cell imaging were purchased

312 from available suppliers, or were synthesized as previously described ${ }^{25,26}$.

\section{Protein purification}

315 Proteins were expressed using a pET51b(+) (Novagen) in Escherichia coli BL21 (DE3)

316 pLysS, in the presence of $100 \mu \mathrm{g} \mathrm{mL}^{-1}$ ampicillin in Luria-Bertani, shaking at $220 \mathrm{rpm}$. 
317 Cultures were grown at $37^{\circ} \mathrm{C}$ until an $\mathrm{OD}_{600}$ of 0.8 was reached, and then induced

318 with $1 \mathrm{mM}$ isopropyl $\beta$-thiogalacopyranoside (ITPG). After overnight expression at 25

$319{ }^{\circ} \mathrm{C}$, cells were harvested and lysed by sonication. The lysates were cleared by centrifugation and purified by IMAC using Ni-NTA Resin (Thermo Fisher Scientific).

321 For proteins used in TR-FRET assays, His-tag purification was followed by Strep-

322 Tactin purifications (IBA Lifesciences), according to the manufacturer's protocol.

\section{Fluorescence emission titrations}

wtGFP emission assays were performed in $50 \mathrm{mM}$ HEPES, $50 \mathrm{mM} \mathrm{NaCl}, 0.5$ mg mL-

1 BSA, 0.05\% Triton-X 100, pH 7.3, in 384-well plates (Black, flat-bottom, Corning \#3821). Dilution series of the protein switch in the presence of TMP (500 $\mu \mathrm{M}$, final and incubated at room temperature for 10 minutes. wtGFP (10 nM, final concentration) was diluted in $50 \mathrm{mM}$ HEPES, $50 \mathrm{mM} \mathrm{NaCl}, 0.5 \mathrm{mg} \mathrm{mL}^{-1} \mathrm{BSA}, 0.05 \%$ Triton-X 100, $\mathrm{pH}$ 7.3. Protein were then mixed in a 1:1 ratio in the plate, and the plate read in a Spark ® 20M microplate reader (Tecan). Excitation wavelength was $470 \mathrm{~nm}(5 \mathrm{~nm}$ bandwidth). Emission wavelength was $535 \mathrm{~nm}$ ( $5 \mathrm{~nm}$ bandwidth). The titration curves were fit with the full equation of single site binding, accounting for the effect of nonspecific binding 27 .

\section{TR-FRET assay}

338 Constructs assayed by TR-FRET were expressed as SNAP-tag fusions and EGFPfusions. SNAP-tag on the target proteins $(4 \mu \mathrm{M})$ was labeled with excess of SNAP-

340 Lumi4-Tb (Cisbio) $(6 \mu \mathrm{M})$ in $50 \mathrm{mM}$ HEPES, $50 \mathrm{mM} \mathrm{NaCl}$, pH 7.3, at room temperature 
341 for 4 hours. Excess unlabeled dye was removed by centrifugal filter units (Amicon).

342 Tb-labeled target protein was diluted in $50 \mathrm{mM}$ HEPES, $50 \mathrm{mM} \mathrm{NaCl}, 0.5 \mathrm{mg} \mathrm{mL}^{-1}$

343 BSA, $0.05 \%$ Triton-X 100, pH 7.3, containing $100 \mu \mathrm{M}$ NADPH and placed into 384-

344 well plates (Black, flat-bottom, Corning \#3821). EGFP-fused proteins were diluted in $50 \mathrm{mM}$ HEPES, $50 \mathrm{mM} \mathrm{NaCl}, 0.5 \mathrm{mg} \mathrm{mL}^{-1} \mathrm{BSA}, 0.05 \%$ Triton-X 100 in the presence of TMP (500 $\mu \mathrm{M}$, final concentration) or DMSO and/or NADPH (100 $\mu \mathrm{M}$, final concentration). EGFP-fused proteins were mixed in a 1:1 ratio in the plate with the Tblabeled target and incubated at least $15 \mathrm{~min}$ at room temperature. The plate was then read by a Spark ${ }^{\circledR} 20 \mathrm{M}$ microplate reader (Tecan) in TR-FRET mode. The excitation wavelength was $320 \mathrm{~nm}$ ( $25 \mathrm{~nm}$ bandwidth). The emission wavelength for Tb was 480 $\mathrm{nm}$ (7.5 nm bandwidth), and the emission wavelength for EGFP was $520 \mathrm{~nm}(7.5 \mathrm{~nm}$ $\mu \mathrm{s}$. The titration curves were fit the full equation of single site binding, accounting for the effect of nonspecific binding.

\section{Kinetics of in vitro dissociation}

wtGFP was mixed with an excess of LAMA (1:3 ratio) on ice for 10 min and passed over size-exclusion chromatography. The heterodimeric fraction was collected and diluted into $50 \mathrm{mM}$ HEPES, $50 \mathrm{mM} \mathrm{NaCl}, 0.5 \mathrm{mg} \mathrm{mL}^{-1} \mathrm{BSA}, 0.05 \%$ Triton-X 100 , pH 7.3, in 96-well black flat-bottomed plates to a final concentration of $200 \mathrm{nM}$, in the presence of $100 \mu \mathrm{M}$ NADPH. TMP, Pyr or MTX were diluted in DMSO. $1 \mu \mathrm{L}$ of relevant drug solutions was added to the 96-well plate and fluorescence emission recorded over time on a Spark § $20 \mathrm{M}$ microplate reader (Tecan). Excitation wavelength was $470 \mathrm{~nm}$ ( $5 \mathrm{~nm}$ bandwidth). Emission wavelength was $535 \mathrm{~nm}$ ( $5 \mathrm{~nm}$ bandwidth). For 
reversible association eDHFR was diluted in $50 \mathrm{mM}$ HEPES, $50 \mathrm{mM} \mathrm{NaCl}, 0.5 \mathrm{mg} \mathrm{mL}-$

${ }^{1} \mathrm{BSA}, 0.05 \%$ Triton-X 100 and $1 \mu \mathrm{L}$ added to the reaction mix in the 96-well plate.

The curves where fit with one-phase dissociation models to estimate the half time at these concentrations.

\section{Protein crystallization}

371 For X-ray crystallography, the LAMAs were sub-cloned into a vector carrying an N-

372 terminal Hisx10-tag, followed by a tobacco etch virus (TEV) protease cleavage tag sequence. The production and IMAC purification of the TEV protease was performed as previously described ${ }^{28}$. The His-Tag was removed from the LAMAs by TEV protease cleavage at $30{ }^{\circ} \mathrm{C}$ overnight, at a ratio of 1:20 (TEV protease: LAMA). The digested protein was purified using a reverse IMAC purification method by NTA resin, collecting the flow-through. The protein was passed over a size-exclusion column and concentrated using centrifugal filter units (Amicon). The protein was flash-frozen and stored at $-70{ }^{\circ} \mathrm{C}$. Purified protein in $25 \mathrm{mM}$ HEPES, $25 \mathrm{mM} \mathrm{NaCl}$, pH 7.3 was premixed with NADPH (10 eq) and TMP (10 eq) as solid powders in $300 \mu \mathrm{L}$ volume. The solution was left on ice for 10 minutes before centrifugation (20 000 g, $10 \mathrm{~min}, 4^{\circ} \mathrm{C}$ ) to remove any precipitation.

Crystallization was performed at $20^{\circ} \mathrm{C}$ using the vapor-diffusion method. Crystals of

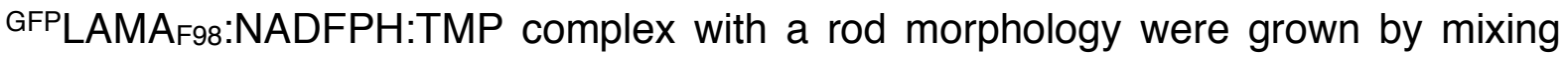
equal volumes of protein solution at $25 \mathrm{mg} / \mathrm{ml}$ in $25 \mathrm{mM}$ HEPES, $25 \mathrm{mM}$ sodium chloride $\mathrm{pH} 7.3$ and a reservoir solution containing $0.1 \mathrm{M} \mathrm{MES} \mathrm{pH} \mathrm{6.0,30 \%} \mathrm{(v/v)} \mathrm{PEG}$ 600, 5\% (w/v) PEG 1000 and 10\% (v/v) glycerol. The crystals were briefly washed in 
cryoprotectant solution consisting of the reservoir solution with sucrose and glucose added to a final concentration of $10 \%(w / v)$ each, prior to flash-cooling in liquid nitrogen. GFPLAMAG97 :NADPH:TMP complex crystals were obtained by mixing equal volumes of protein solution at $15 \mathrm{mg} / \mathrm{ml}$ in $25 \mathrm{mM}$ HEPES, $25 \mathrm{mM}$ sodium chloride $\mathrm{pH}$ be isolated and were briefly washed in cryoprotectant solution consisting of the reservoir solution supplemented with $20 \%(\mathrm{v} / \mathrm{v})$ glycerol before flash-cooling in liquid nitrogen.

\section{X-ray diffraction data collection and structure determination}

400 Single crystal X-ray diffraction data were collected at $100 \mathrm{~K}$ on the X10SA beamline 401 at the SLS (PSI, Villigen, Switzerland). All data were processed with XDS ${ }^{29}$. The 402 structures were determined by molecular replacement (MR) using Phaser ${ }^{30}$ and 403 individual protein coordinates from PDB entries 5UII and 5H8D as a search models 404 for DHFR and nanobody, respectively. The final models were optimized in iterative cycles of manual rebuilding using $\operatorname{Coot}^{31}$ and refinement using $\operatorname{Refmac5}^{32}$ and phenix.refine ${ }^{33}$. Data collection and refinement statistics are summarized in

407 Supplementary Table 1, model quality was validated with MolProbity ${ }^{34}$ as implemented in PHENIX. The omit maps for ligands were generated using the composite omit map 409 tool in PHENIX ${ }^{33}$.

410 Atomic coordinates and structure factors have been deposited in the Protein Data

411 Bank under accession codes: 6RUL (GFPLAMAf98), 6RUM (GFPLAMAG97). Analysis was 412 performed using MacPyMOL ${ }^{35}$ and Coot $^{31}$. 


\section{Mammalian cell culture maintenance}

414 Eukaryotic cells were obtained from American Type Culture Collection (ATCC,

415 Manassas, Virginia), The Leibniz Institute DSMZ-German Collection of

416 Microorganisms and Cell Cultures (DSMZ, Germany), or from collaborators as

417 indicated. No cell lines on the ICLAC list of commonly misidentified cells were used in

418 this work. All cells were cultured in DMEM GlutaMax (Thermo Fisher Scientific)

419 medium supplemented with 10\% FBS, and penicillin and streptomycin as indicated, at

$42037^{\circ} \mathrm{C}$ in a humidified incubator with $5 \% \mathrm{CO}_{2}$. All cells were mycoplasma-free.

421

\section{Generation of ${ }^{\text {p24 }}{ }^{2}$ AMAs98 cell lines}

423 Lentiviral particles were generated by co-transfection of transfer plasmid pWPI EGFP-

424 p24LAMAs98 IRES puro, packaging construct psPAX2, fusion protein expression

425 plasmid pCMV-VSVG and pAdvantage (Promega) in a ratio of $1.5: 1: 0.5: 0.2$, into 426 HEK293T (ATCC) cells using PEI (1:3 ratio of $\mu \mathrm{g}$ DNA : $\mu l 1 \mathrm{mg} / \mathrm{ml}$ PEI). The medium

427 was changed after 6 hours and production of lentiviral particles was allowed to proceed 428 for $48 \mathrm{~h}$. The supernatant was filtered through $0.45 \mu \mathrm{m}$ MCE filters and was directly 429 added to HeLa TZM-bl cells (NIH AIDS Repository). After 2 days the cells were expanded and $1 \mu \mathrm{g} / \mathrm{ml}$ puromycin was added to select for stably transduced cells.

\section{Genome editing}

433 Nup62 in HeLa Kyoto cells was endogenously tagged with mEGFP at the C-terminus by CRISPR-Cas9 nickases and its homozygous integration was validated as described previously ${ }^{36,37}$. The gRNA sequences for the genome editing are as follows: 
438 Previously described genome edited HeLa Kyoto Mad2L1-EGFP ${ }^{20}$ were used in the 439 generation of stable cell lines expressing LAMAs. HeLa Kyoto Mad2L1-EGFP cells 440 were seeded one day before transfection with Lipofectamin3000 (Thermo Fisher 441 Scientific), in the presence of TMP $(10-50 \mu \mathrm{M})$, following the manufacturer's 442 instructions. Cells were seeded monoclonal densities and selected using 500-800 $\mu \mathrm{g}$ $443 \mathrm{~mL}^{-1}$ geneticin (Thermo Fisher Scientific), and TMP (10-50 $\left.\mu \mathrm{M}\right)$ for 2 weeks. Cells were 444 then labeled with BG-SiR (500 nM) overnight, before sorting on a FACSMelody (BD biosciences) for LAMA expressing cells.

\section{Live cell imaging of ${ }^{24}$ LAMAs98 in HIV expressing cells}

HeLa TZM-bl cells were seeded the day before transfection in complete medium with $100 \mathrm{U} / \mathrm{ml}$ penicillin $100 \mu \mathrm{g} / \mathrm{ml}$ streptomycin and incubated at $37{ }^{\circ} \mathrm{C}$ and $5 \% \mathrm{CO}_{2}$. pCHIV env(stop) was transfected in a 1:1 ratio with pCHIV env(stop) gag-mCherry using Turbofect (1:2 ratio). The cells were incubated for 24 hours, the medium was changed to imaging medium (FluoroBrite DMEM (Thermo Fisher Scientific), $10 \%$ FBS, 4 mM GlutaMAX (Gibco Life Technologies), 2 mM sodium pyruvate (Gibco Life Technologies), 20 mM HEPES pH 7.4, 100 U/ml Penicillin $100 \mu \mathrm{g} / \mathrm{ml}$ Streptomycin (PAN-Biotech, Germany)) and transferred to a Nikon Eclipse Ti2 (Nikon, Japan)

457 inverted microscope equipped with an Andor confocal spinning disc unit (Yokogawa 458 CSU-W1 Spinning Disk Unit, Andor, Oxford Instruments, United Kingdom). Cells were 459 imaged at $37^{\circ} \mathrm{C}$ and $5 \% \mathrm{CO}_{2}$ using a $100 \times$ oil-immersion objective (Nikon CFI 460 Apochromat TIRF 100X Oil NA 1.49) and a dual EMCCD camera setup (ANDOR iXon 
DU-888), simultaneously recording the EGFP (488/500-550 nm) and the mCherry channel $(568 / 575-625 \mathrm{~nm})$ with a pixel size of $0.13 \mu \mathrm{m}$. 3D stacks $(0.5 \mu \mathrm{m}, \mathrm{z}$-spacing) were recorded with a time interval of 3 minutes for $60-120$ minutes at up to 32 randomly chosen positions using the Nikon Imaging Software Elements 5.02. After 4-12 frames $50 \mu$ TMP containing imaging medium was added to a final concentration of $10 \mu \mathrm{M}$ and imaging was continued. The movies were filtered in Fiji/lmageJ with a mean filter (kernel size: $0.25 \times 0.25 \mu \mathrm{m}$ ) to reduce noise and the mean intensity of a region of interest inside the nucleus was measured using the Multi Measure function of the ROI

Manager. Camera background was subtracted and intensities where normalized to the highest intensity within the ROI during the timeseries to correct for different expression levels. Different experiments were temporally aligned to the time of TMP addition and data was pooled from 3 independent experiments.

\section{Translocation of fluorescent proteins}

HeLa Kyoto cells ${ }^{36}$ were seeded 24 hours before transfection with Lipofectamin2000

(Thermo Fisher Scientific) according to the manufacturer's protocol. After 24 hours,

477 complete medium without phenol red was added to the cells. Cells were labeled with

BG-SiR (500 nM) overnight, in the presence or absence of $10 \mu \mathrm{M}$ TMP or DMSO,

479 before being imaged by confocal microscopy using a Leica DMi8 microscope (Leica

481 light laser, and an HC PL APO 40x/1.10 W motCORR CS2 objective, at $37^{\circ} \mathrm{C}$ with 5 $482 \% \mathrm{CO}_{2}$, achieved by a temperature controllable incubator (Life Imaging Services). 483 Image acquisition was performed with speed of $400 \mathrm{~Hz}$, pixel dwell time $600 \mathrm{~ns}$, pixel 484 size $0.06 \mu \mathrm{m}$, with z-stacks of $1 \mu \mathrm{m}$ over $10 \mu \mathrm{m}$. A white-light laser was used for 
excitation, collecting with Leica HyD detectors: EGFP (488/505-550 nm), YFP (514/525-573 nm), ShadowG-mScarlet (561/583-625 nm), SiR (633/650-750 nm).

\section{Perfusion of TMP over live cells}

U-2 OS (DSMZ) cells were transfected with Lipofectamin2000 (Thermo Fisher Scientific) according to the manufacturer's protocol. After 24 hours, cells were seeded on Ibidi 0.6 Luer I cell culture treated perfusion chambers. After the cells were adherent, BG-SiR (500 nM) was added to the perfusion chamber and labeled overnight at $37^{\circ} \mathrm{C}$. The perfusion chamber was then attached to a custom-built gravityperfusion system and mounted on a Leica DMi8 microscope (Leica Microsystems, Germany) equipped with a Leica TCS SP8 X scanhead; a SuperK white light laser, and an HC PL APO 40×/1.10 W motCORR CS2 objective, at $37^{\circ} \mathrm{C}$. TMP $(0.5 \mu \mathrm{M}-20$ $\mu \mathrm{M})$ in complete DMEM GlutMax medium with phenol red was perfused over the cells, and images acquired at a scanning speed of $400 \mathrm{~Hz}$, pixel dwell time $1.2 \mu \mathrm{s}$, with a pinhole at 1 airy unit, with z-stacks of $1 \mu \mathrm{m}$ over $10-20 \mu \mathrm{m}$, with image acquisition every 30-60 s. A white-light laser was used for excitation, collecting with Leica HyD detectors: EGFP (488/505-550 nm), SiR (633/650-750 nm). Image analysis was performed in Fiji/ImageJ using the Time Series Analyzer (3.0), selecting a ROI of interest in the nucleus, and measuring the fluorescent intensity over time, with background subtraction of a region outside of the cells. All values were normalised to the intensity in the nucleus after the highest concentration of TMP perfused for each cell. Different experiments were temporally aligned to the time of TMP addition and data was pooled from 3 independent experiments. 


\section{Live-cell imaging of Nup62-mEGFP}

510 Live-cell imaging of HeLa Nup62-mEGFP cells was performed at $37{ }^{\circ} \mathrm{C}$ in $\mathrm{CO}_{2}-$

511 independent medium without phenol red (Invitrogen, Carlsbad, CA) containing 20\%

512 FBS, $2 \mathrm{mM}$ l-glutamine, and $100 \mu \mathrm{g} / \mathrm{ml}$ penicillin and streptomycin, with either $10 \mu \mathrm{M}$

513 of TMP or DMSO. Cells were incubated with $10 \mu \mathrm{M}$ BG-TMR for 30 min and the BG-

514 TMR was washed away before imaging. Cells were then observed by confocal

515 microscopy (LSM780; Carl Zeiss, Oberkochen, Germany) using a $63 \times 1.4$ NA Plan-

516 Apochromat objective (Carl Zeiss), recording the mEGFP (488/491-552 nm) and TMR

$517(561 / 580-660 \mathrm{~nm})$ channels with a xy resolution of $0.13 \mu \mathrm{m}$ and the section thickness

518 of $1.2 \mu \mathrm{m}$. Fluorescence images were filtered with a median filter (kernel size: $0.25 \times$

$5190.25 \mu \mathrm{m})$ for presentation purposes.

520

521 Automated microscopy and analysis

522 For continuous live-cell imaging of HeLa Kyoto Mad2L1-EGFP cells stably expressing

523 LAMAs, cells were seeded in 96-well plates (Eppendorf), in the presence of TMP (50

$524 \mu \mathrm{M})$. After cells were adherent, cells were labeled with BG-SiR (100 nM) overnight.

525 The cells were then labeled with Hoechst $33342(1 \mu \mathrm{g} / \mathrm{mL})$ in complete medium

526 without phenol red in the presence or absence of TMP $(50 \mu \mathrm{M})$ for $15 \mathrm{~min}$, and washed

5273 times with complete medium without phenol red in the presence or absence of TMP

$528(50 \mu \mathrm{M})$. Cells were imaged in the presence of BG-SiR $(100 \mathrm{nM})$, in the presence or

529 absence of TMP $(50 \mu \mathrm{M})$, and in the presence of additional mitotic drugs as indicated,

530 nocodazole $(330 \mathrm{nM})$ or reversine $(5 \mu \mathrm{M})$. Automatic microscopy was performed with

531 Leica HCS A Matrix Screener software on a Leica DMi8 microscope (Leica

532 Microsystems, Germany) equipped with a Leica TCS SP8 X scanhead; a SuperK white 
light laser, and an HC PL APO 40x/1.10 W motCORR CS2 objective, at $37^{\circ} \mathrm{C}, 5 \%$

$\mathrm{CO}_{2}$, achieved by a temperature controllable incubator (Life Imaging Services). A

white-light laser or $405 \mathrm{~nm}$ diode was used to excite the fluorophores, collecting with

Leica HyD detectors: Hoechst (405/425-475 nm), SiR (633/650-750 nm). Image

acquisition was performed with speed of $400 \mathrm{~Hz}$, pixel dwell time $1.2 \mu \mathrm{s}$, pixel size

$0.57 \mu \mathrm{m}$, with z-stacks of $1 \mu \mathrm{m}$ over $10 \mu \mathrm{m}$. Image analysis was performed in

Fiji/lmageJ, with manual annotations of LAMA expressing cells followed from

prometaphase to mitotic exit.

\section{Additional References}

543

544

545

546

547

548

549

550

551

552

553

554

555

556

557

558

559

560

561

562

563

564

565

566

567

568

569

21 Müller, B. et al. Construction and characterization of a fluorescently labeled infectious human immunodeficiency virus type 1 derivative. J. Virol. 78, 1080310813, (2004).

22 Lampe, M. et al. Double-labelled HIV-1 particles for study of virus-cell interaction. Virology 360, 92-104, doi: (2007).

23 Hendrix, J. et al. Live-cell observation of cytosolic HIV-1 assembly onset reveals RNA-interacting Gag oligomers. J. Cell Biol. 210, 629-646, doi:10.1083/jcb.201504006 (2015).

24 Trotard, M. et al. Sensing of HIV-1 Infection in Tzm-bl Cells with Reconstituted Expression of STING. J. Virol. 90, 2064-2076, (2016).

25 Lukinavičius, G. et al. A near-infrared fluorophore for live-cell super-resolution microscopy of cellular proteins. Nat. Chem. 5, 132-139, (2013).

26 Keppler, A., Pick, H., Arrivoli, C., Vogel, H. \& Johnsson, K. Labeling of fusion proteins with synthetic fluorophores in live cells. Proc. Natl. Acad. Sci. U.S.A. 101, 9955-9959, (2004).

27 Roehrl, M. H. A., Wang, J. Y. \& Wagner, G. A General Framework for Development and Data Analysis of Competitive High-Throughput Screens for Small-Molecule Inhibitors of Protein-Protein Interactions by Fluorescence Polarization. Biochemistry 43, 16056-16066, (2004).

28 Cabrita, L. D. et al. Enhancing the stability and solubility of TEV protease using in silico design. Protein Sci. 16, 2360-2367, (2009).

29 Kabsch, W. XDS. Acta. Crystallogr. D Biol. Crystallogr. 66, 125-132, (2010).

30 McCoy, A. J. et al. Phaser crystallographic software. J. Appl. Crystallogr. 40, 658-674, (2007).

31 Emsley, P., Lohkamp, B., Scott, W. G. \& Cowtan, K. Features and development of Coot. Acta. Crystallogr. D Biol. Crystallogr. 66, 486-501, (2010). 
$57032 \quad$ Murshudov, G. N. et al. REFMAC5 for the refinement of macromolecular crystal structures. Acta. Crystallogr. D Biol. Crystallogr. 67, 355-367, (2011). Adams, P. D. et al. PHENIX: a comprehensive Python-based system for macromolecular structure solution. Acta. Crystallogr. D Biol. Crystallogr. 66, 213-221, (2010).

57534 Chen, V. B. et al. MolProbity: all-atom structure validation for macromolecular crystallography. Acta. Crystallogr. D Biol. Crystallogr. 66, 12-21, (2010).

35 DeLano, W. L. Pymol: An open-source molecular graphics tool. CCP4 Newsletter On Protein. Crystallography 40, 82-92 (2002).

36 Koch, B. et al. Generation and validation of homozygous fluorescent knock-in cells using CRISPR-Cas9 genome editing. Nat. Protocols 13, 1465-1487, (2018).

37 Otsuka, S. et al. Postmitotic nuclear pore assembly proceeds by radial dilation of small membrane openings. Nat. Struct. Mol. Biol. 25, 21-28, (2018). 